\title{
Eosinophil-to-monocyte ratio is a potential biomarker in the prediction of functional outcome among patients with acute ischemic stroke
}

Shuhong Yu ${ }^{1 \dagger}$, Yi Luo ${ }^{1 \dagger}$, Tan Zhang ${ }^{1}$, Chenrong Huang ${ }^{1}$, Yu Fu' , Qiang Zhang ${ }^{1}$, Fangyue Zeng ${ }^{1}$, Hao Huang ${ }^{1}$, Chunyuan Zhang ${ }^{2^{*}}$ and Zhiliang Guo ${ }^{2^{*}}$ (D)

\begin{abstract}
Background: It has been shown that eosinophils are decreased and monocytes are elevated in patients with acute ischemic stroke (AIS), but the impact of eosinophil-to-monocyte ratio (EMR) on clinical outcomes among AIS patients remains unclear. We aimed to determine the relationship between EMR on admission and 3-month poor functional outcome in AIS patients.

Methods: A total of 521 consecutive patients admitted to our hospital within $24 \mathrm{~h}$ after onset of AIS were prospectively enrolled and categorized in terms of quartiles of EMR on admission between August 2016 and September 2018. The endpoint was the poor outcome defined as modified Rankin Scale score of 3 to 6 at month 3 after admission.

Results: As EMR decreased, the risk of poor outcome increased ( $p<0.001)$. Logistic regression analysis revealed that EMR was independently associated with poor outcome after adjusting potential confounders (odds ratio, 0.09; 95\% $\mathrm{Cl} 0.03-0.34 ; p=0.0003$ ), which is consistent with the result of EMR (quartile) as a categorical variable (odds ratio, 0.23 ; 95\% Cl 0.10-0.52; $\left.p_{\text {trend }}<0.0001\right)$. A non-linear relationship was detected between EMR and poor outcome, whose point was 0.28 . Subgroup analyses further confirmed these associations. The addition of EMR to conventional risk factors improved the predictive power for poor outcome (net reclassification improvement: $2.61 \%, p=0.382$; integrated discrimination improvement: $2.41 \%, p<0.001$ ).
\end{abstract}

Conclusions: EMR on admission was independently correlated with poor outcome in AIS patients, suggesting that EMR may be a potential prognostic biomarker for AIS.

Keywords: Eosinophil-to-monocyte ratio (EMR), Ischemic stroke, Outcome, Biomarker

*Correspondence: zcyself@sina.com; guozhiliang3@163.com

${ }^{\dagger}$ Shuhong Yu and Yi Luo contributed equally to this work

${ }^{2}$ Department of Neurology and Suzhou Clinical Research Center of Neurological Disease, The Second Affiliated Hospital of Soochow University, No. 1055, Sanxiang Road, Suzhou 215004, Jiangsu, China Full list of author information is available at the end of the article

\section{Background}

Inflammatory and immunological responses play pivotal roles in the pathogenesis of acute ischemic stroke (AIS) which is still a main challenge to public health [1-3]. Leukocytes including monocytes, neutrophils, and lymphocytes are indispensable inflammatory cells and are associated with endothelial dysfunction, thrombosis, blood-brain barrier disruption and tissue damage in AIS [1-4]. Moreover, eosinophils have also

(c) The Author(s) 2021. This article is licensed under a Creative Commons Attribution 4.0 International License, which permits use, sharing, adaptation, distribution and reproduction in any medium or format, as long as you give appropriate credit to the original author(s) and the source, provide a link to the Creative Commons licence, and indicate if changes were made. The images or other third party material in this article are included in the article's Creative Commons licence, unless indicated otherwise in a credit line to the material. If material is not included in the article's Creative Commons licence and your intended use is not permitted by statutory regulation or exceeds the permitted use, you will need to obtain permission directly from the copyright holder. To view a copy of this licence, visit http://creativeco mmons.org/licenses/by/4.0/. The Creative Commons Public Domain Dedication waiver (http://creativecommons.org/publicdomain/ zero/1.0/) applies to the data made available in this article, unless otherwise stated in a credit line to the data. 
been shown to be inflammatory cells and were able to regulate the inflammatory responses by facilitating the resolution of inflammation [5]. Recent studies have discovered that stroke triggers an acute decrease in circulating eosinophil counts and an increase in circulating monocytes [6]. Furthermore, post-stroke low circulating eosinophil count was inversely associated with stroke severity and risk of mortality, and high peripheral blood monocyte level was associated with high risk of poor outcome after stroke $[7,8]$. Since the single inflammatory cell is unable to summarize the overall systematic inflammation, new indexes by combining different subtypes of the leukocyte are needed [3, 9]. Given the deleterious effects of classical monocytes and the possible neuroprotective effect of eosinophils in stroke [5, 10-12], eosinophil-to-monocyte ratio (EMR), a novel biomarker reflecting the integrated application value of eosinophils and monocytes, is needed to identify patients at high risk of poor prognosis.

In this study, we aimed to determine the relationship between EMR on admission and 3-month poor functional outcome, and to explore the predictive value of EMR in the poor functional outcome in patients with AIS.

\section{Methods}

\section{Study population}

Consecutive patients with ischemic stroke admitted to the department of encephalopathy at our hospital from August 2016 to September 2018 were prospectively recruited. The inclusion criteria for enrollment were as follows: (1) diagnosis of AIS according to the World Health Organization criteria based on patient history, clinical data, and neuroimaging results (computed tomography or magnetic resonance imaging) [13]; (2) time from onset of stroke to hospitalization was $<24 \mathrm{~h}$; and (3) the patient or their relatives provided informed consent. Study exclusion criteria were: (1) asthma, eosinophilic esophagitis, hypereosinophilic syndrome, evidence of active infection, chronic inflammatory, autoimmune diseases, steroid therapy, cancer, blood system diseases, previous stroke with partial recovery, severe hepatic or renal dysfunction (90 patients); (2) unavailable complete blood cell count or medical records (48 patients); (3) lost to follow-up ( $n=29)$. At last, 521 consecutive ischemic stroke patients were included in the current study. (flowchart of participants selection: Additional file 1: Fig. S1). The study protocol was approved by the institutional Human Research Ethics Committees of Suzhou Integrated Traditional and Western Medicine Hospital, and written informed consent was obtained from all participants or their authorized relatives.

\section{Clinical protocol and laboratory tests}

Medical history including potential stroke risk factors, clinical examination, blood tests, 12-lead electrocardiogram and treatment administration were performed at admission. Stroke severity was assessed by a certified neurologist using the National Institutes of Health Stroke Scale (NIHSS) at admission. The etiologic subtypes of ischemic stroke were classified according to the TOAST (Trial of ORG 10172 in Acute Stroke Treatment) criteria [14]. Given the relatively small number of participants in the other determined etiology and unknown subtypes, we combined these two groups into one group (other etiology/unknown).

In all patients, peripheral venous blood samples were obtained for the measurement of leucocytes, the value of EMR and measuring serum lipid levels. EMR was calculated by dividing eosinophil count by monocyte count. The follow-up data were achieved by telephone. The endpoint was the poor outcome at month 3 after admission with a modified Rankin Scale (mRS) score of 3-6.

\section{Statistical analysis}

The total procedure of statistical analysis was divided into five steps. First, baseline characteristics of study participants were presented according to the quartiles of EMR. The one-way ANOVA (normal distribution), Kruskal-Wallis $\mathrm{H}$ (skewed distribution) test and chisquare test (categorical variables) were used to determine any significant differences between groups according to the quartiles of EMR. Second, we used a univariate regression model to evaluate the associations between EMR and 3-month poor outcome in patients with AIS. For multivariate analysis, we first included age and sex (model 1) and then included variables in the final models if they were significantly associated with poor outcome $(p<0.10)$ or changed the estimates of EMR on poor outcome by more than $10 \%$ (model 2; age, sex, history of hyperlipidemia, history of previous stroke, history of atrial fibrillation, ischemic stroke subtypes, triglyceride, NIHSS score at baseline, premorbid mRS score and proton pump inhibitors treatment). Additional file 1: Tables S2-S5 show the associations of each confounder with the outcomes of interest $[15,16]$. Third, we used generalized additive models (GAM) to identify the non-linear relationships because EMR was a continuous variable. If a non-linear relationship was observed, a two-piecewise linear regression model was used to calculate the threshold effect of the EMR on poor outcome in terms of the smoothing plot. When the ratio between EMR and poor outcome appeared obvious in a smoothed curve, the recursive method automatically calculates the inflection point, where the maximum model likelihood will be used 
[15]. Fourth, we conducted subgroup analyses to assess the robustness of association between low EMR and poor outcome of AIS by using of stratified linear regression models. The modifications and interactions between EMR and subgroup variables on the poor outcome were tested by likelihood ration tests [16]. Fifth, receiver operating characteristic (ROC) curves were used to test the overall discriminative ability of the EMR, eosinophils and monocytes for poor outcome. The differences in discriminative ability were tested using the DeLong method [17]. Moreover, we constructed a conventional model (only including conventional risk factors: age, sex, history of hyperlipidemia, history of previous stroke, history of atrial fibrillation, ischemic stroke subtypes, triglyceride and NIHSS score at baseline) and a new model (including conventional risk factors and EMR) by logistic regression model. To assess the improvement in risk prediction for poor prognosis of ischemic stroke by adding EMR to conventional risk factors, we calculated net reclassification improvement (NRI) and integrated discrimination improvement (IDI) through comparing these 2 models.
All analyses were performed with the statistical software package R (http://www.R-project.org, The R Foundation) and EmpowerStats (http://www.empowerstats.com, X\&Y Solutions, Inc., Boston, MA). A two-sided $p$ value $<0.05$ was considered to be statistically significant [15].

\section{Results}

\section{Baseline characteristics of patients}

Most of the baseline characteristics were balanced between patients included and patients excluded (Additional file 1: Table S1). A total of five hundred and twentyone patients with ischemic stroke were included in the current study and the average age was 69 years. Main baseline characteristics of study participants according to quartiles of EMR are presented in Table 1. The participants with lower EMR values tended to be older, female and to have subtype of cardioembolism, and had higher NIHSS score, history of hyperlipidemia, higher highdensity lipoprotein cholesterol levels, and lower levels of triglyceride. These patients also were more likely to have poor outcome (Table 1).

Table 1 Baseline characteristics of study participants according to quartiles of eosinophil-to-monocyte ratio (EMR)

\begin{tabular}{|c|c|c|c|c|c|}
\hline Characteristics & Q1 & Q2 & Q3 & Q4 & $p$ value \\
\hline No. of patients & 125 & 135 & 130 & 131 & \\
\hline Age, years, mean (SD) & $72.54(13.50)$ & $70.09(12.48)$ & $67.58(12.64)$ & $67.59(13.96)$ & 0.006 \\
\hline Female, $(\%)$ & $64(51.20 \%)$ & $69(51.11 \%)$ & $50(38.46 \%)$ & $49(37.40 \%)$ & 0.026 \\
\hline Hypertension, (\%) & $113(90.40 \%)$ & 109 (80.74\%) & $113(86.92 \%)$ & $110(83.97 \%)$ & 0.150 \\
\hline Diabetes, \% & $34(27.20 \%)$ & $31(22.96 \%)$ & $34(26.15 \%)$ & $28(21.37 \%)$ & 0.671 \\
\hline Hyperlipidemia, \% & $36(28.80 \%)$ & $53(39.26 \%)$ & $55(42.31 \%)$ & $65(49.62 \%)$ & 0.008 \\
\hline Previous stroke, $\%$ & $26(20.80 \%)$ & $28(20.74 \%)$ & $21(16.15 \%)$ & $21(16.03 \%)$ & 0.594 \\
\hline Coronary heart disease, \% & $16(12.80 \%)$ & $10(7.41 \%)$ & $14(10.77 \%)$ & $11(8.40 \%)$ & 0.461 \\
\hline Atrial fibrillation, $\%$ & $26(20.80 \%)$ & $25(18.52 \%)$ & $19(14.62 \%)$ & $16(12.21 \%)$ & 0.245 \\
\hline Current cigarette smoking, \% & $14(11.20 \%)$ & $8(5.93 \%)$ & $19(14.62 \%)$ & $13(9.92 \%)$ & 0.138 \\
\hline NIHSS, median (IQR) & $4(2-8)$ & $4(2-6)$ & $3(1-6)$ & $2(1-6)$ & 0.005 \\
\hline Premorbid mRS score, median (IQR) & $0(0-0)$ & $0(0-0)$ & $0(0-0)$ & $0(0-0)$ & 0.293 \\
\hline Stroke subtypes & & & & & 0.006 \\
\hline Large artery atherosclerosis & $40(32.00 \%)$ & $61(45.19 \%)$ & $48(36.92 \%)$ & $39(29.77 \%)$ & \\
\hline Cardioembolic stroke & $21(16.80 \%)$ & $22(16.30 \%)$ & $17(13.08 \%)$ & $17(12.98 \%)$ & \\
\hline Small artery disease & $59(47.20 \%)$ & $52(38.52 \%)$ & $64(49.23 \%)$ & $75(57.25 \%)$ & \\
\hline Other etiology/unknown & $5(4.00 \%)$ & $0(0.00 \%)$ & $1(0.77 \%)$ & $0(0.00 \%)$ & \\
\hline IV rtPA, \% & $7(5.60 \%)$ & $13(9.63 \%)$ & $9(6.92 \%)$ & $16(12.21 \%)$ & 0.237 \\
\hline Proton pump inhibitors, $\%$ & $56(44.80 \%)$ & $54(40.00 \%)$ & $59(45.38 \%)$ & $44(33.59 \%)$ & 0.186 \\
\hline Triglyceride, mmol/L, median (IQR) & $1.20(0.89-1.60)$ & $1.38(1.00-1.82)$ & $1.40(0.94-1.92)$ & $1.52(1.06-2.38)$ & 0.005 \\
\hline Total cholesterol, mmol/L, mean (SD) & $4.77(1.06)$ & $4.99(1.18)$ & $4.92(1.37)$ & $5.00(1.04)$ & 0.364 \\
\hline High-density lipoprotein cholesterol, mmol/L, mean (SD) & $1.36(0.35)$ & $1.34(0.39)$ & $1.28(0.37)$ & $1.24(0.37)$ & 0.035 \\
\hline Low-density lipoprotein cholesterol, mmol/L, mean (SD) & $2.37(0.70)$ & $2.52(0.74)$ & $2.48(0.82)$ & $2.53(0.70)$ & 0.290 \\
\hline EMR, median (IQR) & $0.04(0.01-0.05)$ & $0.12(0.10-0.15)$ & $0.26(0.21-0.30)$ & $0.49(0.40-0.76)$ & $<0.001$ \\
\hline Poor outcome & $65(52.00 \%)$ & $53(39.26 \%)$ & $34(26.15 \%)$ & $29(22.14 \%)$ & $<0.001$ \\
\hline
\end{tabular}




\section{Univariate and multivariate analysis}

The results of univariate analysis showed that age, female, history of previous stroke, history of coronary heart disease, history of atrial fibrillation, subtype of cardioembolism, NIHSS score at baseline, premorbid mRS score and proton pump inhibitors treatment were positively correlated with poor outcome, whereas history of hyperlipidemia, triglyceride, subtype of small-artery occlusion and EMR were negatively associated with poor outcome (Table 2).

Table 3 summarizes the results of multivariate logistic regression model. The EMR as a continuous variable was independently associated with a smaller risk of poor outcome with an adjusted odds ratio (OR) of 0.23 [95\% confidence interval (CI) 0.09-0.56] after adjustment for age and sex (model 1) and 0.09 (0.03-0.34) after adjustment for all potential covariates (model 2). For the purpose of sensitivity analysis, we converted the EMR into categorical variable by quartile and calculated $p$ for trend, the OR $(95 \% \mathrm{CI})$ of poor outcome for those in the highest quartile of EMR were $0.23(0.10-0.52)$ compared with patients in the lowest quartile of EMR. The $p$ for trend was $<0.0001$.

\section{The analyses of non-linear relationship}

In the present study, we analyzed the non-linear relationship between EMR and poor outcome (Fig. 1). The result of smooth curve showed that the relationship between EMR and poor outcome was non-linear after adjustment for all potential covariates $(p=0.0008)$. We compared linear regression model (fitting the relationship between EMR and poor outcome by a linear) and two-piecewise linear regression model (fitting the relationship between EMR and poor outcome by a curve) (Table 4). The $p$ for log likelihood ratio test is 0.013 which is less than 0.05 . This result indicates that the two-piecewise linear regression model should be used to fit the relationship between EMR and poor outcome. By using a two-piecewise linear regression model, we calculated the inflection point was 0.28 . On the left of inflection point, the effect size was 0.00 (95\% CI $0.00-0.06, p=0.0002$ ). However, on the right side of the inflection point, we did not observe

Table 2 The results of univariate analysis

\begin{tabular}{|c|c|c|c|}
\hline \multirow[t]{2}{*}{ Characteristics } & \multicolumn{2}{|l|}{ Poor outcome } & \multirow[t]{2}{*}{$p$ value } \\
\hline & No & Yes & \\
\hline Age, years, mean (SD) & $66.90 \pm 12.68$ & $74.17 \pm 13.09$ & $<0.001$ \\
\hline Female, $(\%)$ & $140(41.18 \%)$ & $92(50.83 \%)$ & 0.035 \\
\hline Hypertension, (\%) & $285(83.82 \%)$ & $160(88.40 \%)$ & 0.159 \\
\hline Diabetes, (\%) & $77(22.65 \%)$ & $50(27.62 \%)$ & 0.208 \\
\hline Hyperlipidemia, (\%) & $147(43.24 \%)$ & $62(34.25 \%)$ & 0.046 \\
\hline Previous stroke, (\%) & $49(14.41 \%)$ & $47(25.97 \%)$ & 0.001 \\
\hline Coronary heart disease, (\%) & $20(5.88 \%)$ & $31(17.13 \%)$ & $<0.001$ \\
\hline Atrial fibrillation, (\%) & $29(8.53 \%)$ & $57(31.49 \%)$ & $<0.001$ \\
\hline Current cigarette smoking, (\%) & $33(9.71 \%)$ & $21(11.60 \%)$ & 0.499 \\
\hline NIHSS, median (IQR) & $2(1-3)$ & $7.00(4-11)$ & $<0.001$ \\
\hline Premorbid mRS score, median (IQR) & $0(0-0)$ & $0(0-0)$ & 0.065 \\
\hline Stroke subtypes & & & $<0.001$ \\
\hline Large artery atherosclerosis & $108(31.76 \%)$ & $80(44.20 \%)$ & \\
\hline Cardioembolic stroke & $25(7.35 \%)$ & $52(28.73 \%)$ & \\
\hline Small artery disease & $201(59.12 \%)$ & $49(27.07 \%)$ & \\
\hline Other etiology/unknown & $6(1.76 \%)$ & $0(0.00 \%)$ & \\
\hline IV rtPA, (\%) & $29(8.53 \%)$ & $16(8.84 \%)$ & 0.904 \\
\hline Proton pump inhibitors & $106(31.18 \%)$ & $107(59.12 \%)$ & $<0.001$ \\
\hline Triglyceride, mmol/L, median (IQR) & $1.46(1.02-2.03)$ & $1.21(0.94-1.67)$ & 0.001 \\
\hline Total cholesterol, mmol/L, mean (SD) & $4.91 \pm 1.45$ & $4.94 \pm 1.15$ & 0.765 \\
\hline High-density lipoprotein cholesterol, mmol/L, mean (SD) & $1.29 \pm 0.37$ & $1.33 \pm 0.38$ & 0.289 \\
\hline Low-density lipoprotein cholesterol, mmol/L, mean (SD) & $2.46 \pm 0.74$ & $2.50 \pm 0.74$ & 0.577 \\
\hline EMR, median (IQR) & $0.22(0.10-0.39)$ & $0.12(0.05-0.23)$ & $<0.001$ \\
\hline
\end{tabular}


Table 3 Relationship between eosinophil-to-monocyte ratio (EMR) and poor outcome among patients with acute ischemic stroke in different models

\begin{tabular}{|c|c|c|c|c|c|c|}
\hline \multirow[t]{2}{*}{ Variable } & \multicolumn{2}{|c|}{ Non-adjusted model } & \multicolumn{2}{|l|}{ Model 1} & \multicolumn{2}{|l|}{ Model 2} \\
\hline & OR $(95 \% \mathrm{Cl})$ & $P$ & OR $(95 \% \mathrm{Cl})$ & $P$ & OR $(95 \% \mathrm{Cl})$ & $P$ \\
\hline EMR & $0.19(0.08,0.46)$ & 0.0002 & $0.23(0.09,0.56)$ & 0.0011 & $0.09(0.03,0.34)$ & 0.0003 \\
\hline \multicolumn{7}{|l|}{ EMR (quartile) } \\
\hline Q1 & 1.0 & & 1.0 & & 1.0 & \\
\hline Q2 & $0.60(0.36,0.98)$ & 0.0398 & $0.64(0.38,1.07)$ & 0.0869 & $0.56(0.29,1.10)$ & 0.0903 \\
\hline Q3 & $0.33(0.19,0.55)$ & $<0.0001$ & $0.38(0.22,0.66)$ & 0.0005 & $0.27(0.13,0.59)$ & 0.0010 \\
\hline Q4 & $0.26(0.15,0.45)$ & $<0.0001$ & $0.30(0.17,0.52)$ & $<0.0001$ & $0.23(0.10,0.52)$ & 0.0005 \\
\hline$P$ for trend & $<0.0001$ & & $<0.0001$ & & $<0.0001$ & \\
\hline
\end{tabular}

Non-adjusted model: we did not adjust other covariates

Model 1: we adjusted age and sex

Model 2: we adjusted age, sex, history of hyperlipidemia, history of previous stroke, history of atrial fibrillation, ischemic stroke subtypes, triglyceride, NIHSS score at baseline, premorbid $\mathrm{mRS}$ score and proton pump inhibitors treatment

EMR eosinophil-to-monocyte ratio, OR odds ratio

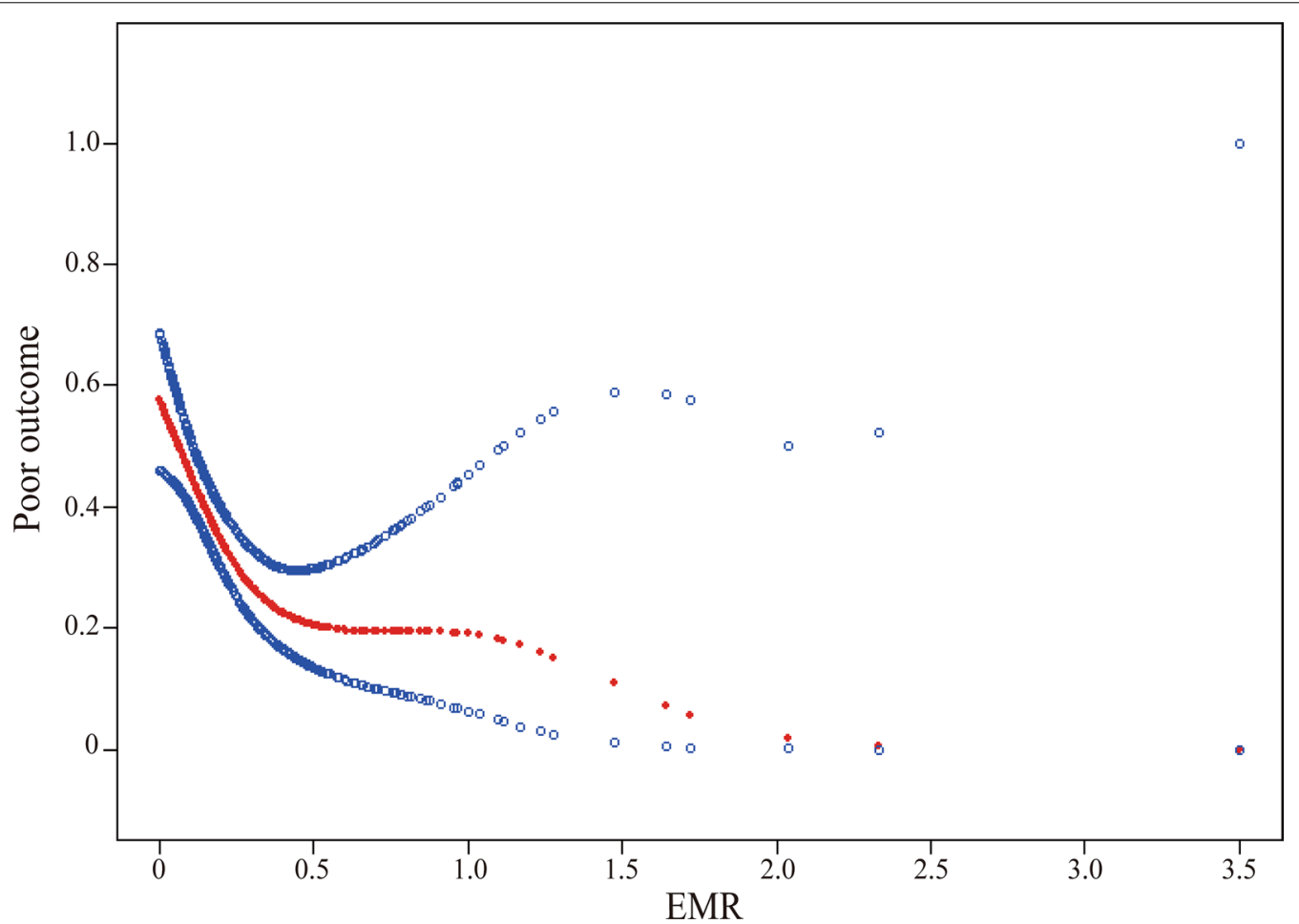

Fig. 1 The non-linear relationship between eosinophil-to-monocyte ratio (EMR) and poor outcome of ischemic stroke. A nonlinear relationship between them was detected after adjusting for age, sex, history of hyperlipidemia, history of previous stroke, history of atrial fibrillation, ischemic stroke subtypes, triglyceride, NIHSS score at baseline, premorbid mRS score and proton pump inhibitors treatment

a significant association between EMR and poor outcome (0.42, 95\% CI 0.09-1.94, $p=0.2656$; Table 4).

\section{The results of subgroup analyses}

As is shown in Table 5, the test of interactions was significant for NIHSS ( $p$ for interaction $=0.0023$ ), while the tests of interactions were not statistically significant for age, sex, history of hypertension, history of diabetes mellitus, history of hyperlipidemia, history of previous stroke, history of coronary heart disease, history of atrial fibrillation, current cigarette smoking, ischemic stroke subtypes, receiving treatment with intravenous 
Table 4 The results of two-piecewise linear regression model

\begin{tabular}{lll}
\hline Outcome: & $\begin{array}{l}\text { Effect size } \\
\text { OR }(\mathbf{9 5} \% \mathbf{C l})\end{array}$ & $\boldsymbol{p}$ value \\
\hline Inflection point of EMR & 0.28 & \\
$<0.28$ & $0.00(0.00,0.06)$ & 0.0002 \\
$\geq 0.28$ & $0.42(0.09,1.94)$ & 0.2656 \\
$p$ for log likelihood ratio test & 0.013 & \\
\hline
\end{tabular}

Effect: EMR; cause: poor outcome; adjusted: age, sex, history of hyperlipidemia, history of previous stroke, history of atrial fibrillation, ischemic stroke subtypes, triglyceride and NIHSS score at baseline

$E M R$ eosinophil-to-monocyte ratio, $O R$ odds ratio

recombinant tissue plasminogen activator and proton pump inhibitors treatment ( $p$ values for interaction were larger than 0.05). The effect sizes of EMR on poor outcome showed significant differences in different NIHSS score. EMR was negatively associated with poor outcome in participants with minor stroke (NIHSS score $<4)(0.00$, $95 \%$ CI $0.00-0.03, p=0.0006)$. However, the effect sizes of EMR on poor outcome was statistically non-significant in participants with NIHSS score $\geq 4(p=0.0789 ; p$ for interaction $=0.0023$ in the model 2 ).

\section{Incremental prognostic value of EMR in patients with ischemic stroke}

ROC curves comparing the discrimination performance of EMR, eosinophil and monocyte count on poor outcome are shown in Additional file 1: Fig. S2. The area under the curve (AUC) of EMR (AUC: 0.6532; 95\% CI 0.6032-0.7031) for poor outcome is greater than those of eosinophils (AUC: 0.6257; 95\% CI 0.5746-0.6769; $p=0.0007$ ) and monocytes (AUC: $0.5831 ; 95 \%$ CI $0.5317-0.6344 ; p=0.0452$ ). In addition, we examined whether adding serum EMR to the conventional risk factors improved the risk prediction of clinical outcomes after AIS. And we found adding EMR to conventional risk factors significantly improved predictive power for poor outcome (NRI: $2.61 \%, p=0.382$; IDI: $2.41 \%, p<0.001$ ).

\section{Discussion}

Previous studies have shown that stroke triggers an acute decrease in circulating eosinophil counts and an increase in circulating monocytes [6]. Furthermore, post-stroke low circulating eosinophil count was inversely associated with stroke severity and risk of mortality, and high peripheral blood monocyte level was associated with high risk of poor outcome after stroke [7, 8]. These studies, however, did not control for stroke severity and have other issues such as its retrospective nature or the small sample size that limit interpretation of the findings. In addition, all of these studies are mainly focused on a single subpopulation of leukocytes, which may not provide a comprehensive study for the eosinophils and monocytes [7, 8]. Given the deleterious effects of classical monocytes and the possible neuroprotective effect of eosinophils in stroke [5, 10-12], EMR, a novel biomarker reflecting the integrated application value of eosinophils and monocytes, is needed to identify patients at high risk of poor prognosis [9]. Like other study in different diseases [9], EMR is used to identify patients at high risk of poor outcome in stroke, and we found the lower EMR on admission was associated with higher risk of 3-month poor functional outcome in patients with AIS, and also proved EMR was of certain value in predicting poor outcome in patients with AIS.

Firstly, we demonstrated that there was a saturating effect on the linear relationship between EMR and poor outcome in the present study. The inflection point we calculated by the recursive algorithm was 0.28 . The result means the negative linear association between EMR and poor outcome is only present in participants with relatively low EMR level. For those with relatively high EMR level, this linear relationship cannot be found. There is no current study to explore the non-linear relationships between EMR and poor outcome, but the previous investigations might give us some clues. A recent study revealed that the adjusted odds ratios reflecting the effect sizes of eosinophils on cerebral infarct volume in the Q4 (the reference group), Q3, Q2 and Q1 group were $1.00,2.416,4.988$ and 50.791, respectively. Similarly, on the effect sizes of eosinophils on poor outcome, the odds ratios in 4 quartiles of eosinophils (from high to low) were $1.00,2.747,4.804$ and 30.2991 , respectively [7]. Moreover, on the effect sizes of monocytes on novel plaque formation, the odds ratios in 4 quartiles of monocytes (from low to high) were 1.00, 1.17, 1.12 and 1.85, respectively [18]. This kind of non-equidistant changes in effect size suggested that there may be a nonlinear relationship between EMR and poor outcome. Given that GAM can handle non-parametric smoothing and has obvious advantages in dealing with non-linear relations, the use of GAM will help us to better discover the real relationships between EMR and poor outcome [15]. Therefore, we use the GAM to clarify their nonlinear relationship in the present study and a nonlinear relationship between EMR and poor outcome was detected after adjusting for potential covariates.

Secondly, we eliminated the confounding effects of the severity of AIS by adjusting baseline NIHSS in the multivariable models and performing the collinearity screening and subgroup analyses. Given that the percentage of eosinophils was negatively correlated with infarct volume and eosinopenia had the potential to predict the severity of AIS $[7,19,20]$, we performed the collinearity screening 
Table 5 Subgroup analyses on the association between eosinophil-to-monocyte ratio (EMR) and poor outcome of ischemic stroke

\begin{tabular}{|c|c|c|c|c|}
\hline Subgroup & No. of participants & OR $(95 \% \mathrm{Cl})$ & $p$ value & $p$ for interaction \\
\hline Age, years & & & & 0.2716 \\
\hline$<65$ & 179 & $0.37(0.04,3.57)$ & 0.3900 & \\
\hline$\geq 65$ & 342 & $0.08(0.02,0.35)$ & 0.0008 & \\
\hline Sex & & & & 0.1422 \\
\hline Male & 289 & $0.08(0.02,0.43)$ & 0.0033 & \\
\hline Female & 232 & $0.05(0.01,0.28)$ & 0.0010 & \\
\hline Hypertension & & & & 0.4943 \\
\hline No & 76 & $35.05(0.00, \operatorname{lnf})$ & 0.9999 & \\
\hline Yes & 445 & $0.08(0.02,0.35)$ & 0.0006 & \\
\hline Diabetes & & & & 0.4333 \\
\hline No & 394 & $0.11(0.03,0.40)$ & 0.0008 & \\
\hline Yes & 127 & $0.00(0.00,0.04)$ & 0.0027 & \\
\hline Hyperlipidemia & & & & 0.8288 \\
\hline No & 312 & $0.13(0.02,0.71)$ & 0.0187 & \\
\hline Yes & 209 & $0.05(0.00,0.62)$ & 0.0202 & \\
\hline Previous stroke & & & & 0.1904 \\
\hline No & 425 & $0.06(0.01,0.26)$ & 0.0002 & \\
\hline Yes & 96 & $0.23(0.01,8.31)$ & 0.4233 & \\
\hline Coronary heart disease & & & & 0.5110 \\
\hline No & 470 & $0.08(0.02,0.32)$ & 0.0004 & \\
\hline Yes & 51 & $\operatorname{lnf}^{a}\left(0.00, \operatorname{Inf}^{a}\right)$ & 0.9997 & \\
\hline Atrial fibrillation & & & & 0.5915 \\
\hline No & 435 & $0.08(0.02,0.34)$ & 0.0008 & \\
\hline Yes & 86 & $0.10(0.01,2.02)$ & 0.1344 & \\
\hline Current cigarette smoking & & & & 0.7324 \\
\hline No & 467 & $0.09(0.02,0.35)$ & 0.0004 & \\
\hline Yes & 54 & $0.00\left(0.00, \ln ^{a}\right)$ & 0.9992 & \\
\hline NIHSS & & & & 0.0023 \\
\hline$<4$ & 295 & $0.00(0.00,0.03)$ & 0.0006 & \\
\hline$\geq 4$ & 226 & $0.37(0.12,1.12)$ & 0.0789 & \\
\hline Stroke subtypes & & & & 0.9674 \\
\hline Large artery atherosclerosis & 188 & $0.13(0.02,0.81)$ & 0.02856 & \\
\hline Cardioembolic stroke & 77 & $0.13(0.01,2.35)$ & 0.1659 & \\
\hline Small artery disease & 250 & $0.06(0.00,0.73)$ & 0.0280 & \\
\hline Other etiology/unknown & 6 & $\operatorname{lnf}^{a}$ & & \\
\hline IV rtPA & & & & 0.7945 \\
\hline No & 476 & $0.09(0.02,0.34)$ & 0.0005 & \\
\hline Yes & 45 & $0.00\left(0.00, \ln f^{a}\right)$ & 0.9993 & \\
\hline Proton pump inhibitors & & & & 0.3518 \\
\hline No & 308 & $0.03(0.00,0.26)$ & 0.0016 & \\
\hline Yes & 213 & $0.19(0.04,0.91)$ & 0.0381 & \\
\hline
\end{tabular}

In the multivariate models, confounding factors, such as age, sex, history of hyperlipidemia, history of previous stroke, history of atrial fibrillation, ischemic stroke subtypes, triglyceride, NIHSS score at baseline, premorbid mRS score and proton pump inhibitors treatment were included unless the variable was used as a stratification variable

EMR eosinophil-to-monocyte ratio, IQR interquartile range, IV rtPA intravenous recombinant tissue plasminogen activator, NIHSS National Institutes of Health Stroke Scale, $O R$ odds ratio

${ }^{a}$ The model failed because of the small sample size 
and found there was not collinearity between EMR and NIHSS (Additional file 1: Table S2). Moreover, we found that the significant association of EMR with poor outcome was independent of the baseline NIHSS, especially in participants with minor stroke (NIHSS score $<4$; $p$ for interaction $=0.0023$ in the model 2) in the multivariate analysis and further subgroup analyses. These results suggested that EMR had additional prognostic value when baseline NIHSS was considered, and we should apply EMR for the prediction of poor outcome in participants with minor stroke. The reason why the effect sizes of EMR on poor outcome showed significant differences in different NIHSS score remains unclear. We hypothesized that the complication of patients with severe stroke and higher NIHSS itself might cause some disruptions to the relationship between EMR and poor outcome. Further studies are needed to investigate this hypothesis.

Thirdly, we also explored whether EMR had greater and additional prognostic value for poor outcome by using of various statistical methods. First, the AUC of EMR for poor outcome is greater than that of eosinophil or monocyte count, suggesting that EMR is superior to only the eosinophil count or monocyte count for distinguishing the occurrence of poor outcome. The reasons for such a superiority of EMR may relate to what the EMR represents. The EMR reflects the balance between eosinophil and monocyte levels, which may be comprehensively summarize the overall systematic inflammation conditions [9]. Second, given that the guide advises the visual representation of the relationship between predicted and observed (not the specific $p$ value) is the best way to evaluate calibration [21], our results suggested EMR could significantly improve the predictive power for poor outcome beyond established traditional risk factors (NRI: 2.61\%; IDI: 2.41\%).Therefore, we hypothesized that serum EMR might be useful in risk stratification of poor outcome among patients with ischemic stroke and could assist the selection of high-risk patients in future clinical practice. If patients have low EMR levels at admission, they may be at high risk of poor outcome and should receive aggressive monitoring and therapeutic interventions.

The mechanisms underlying these observations are not well established, but they seem to be related to the roles of eosinophils and monocytes in ischemic insult. Eosinophils can secrete over 35 cytokines, growth factors and chemokines, including IL-4, IL-13 and vascular endothelial growth factor (VEGF). IL-4 and IL-13 are capable of inducing the activation of the M2 phenotype microglia, which possess neuroprotective properties by facilitating the resolution of inflammation. And VEGF might be neuroprotective by the modulation of angiogenesis $[5,10,11]$. The phenotypes of monocytes include
$\mathrm{CD} 14^{\text {high }} \mathrm{CD} 16^{-}$(classical monocytes), $\mathrm{CD} 14^{\mathrm{dim}} \mathrm{CD} 16^{+}$ and $\mathrm{CD} 14^{\text {high }}{ }^{-} \mathrm{CD} 16^{+}$[12]. It is worth noting that the classical monocytes account for nearly $90 \%$, which have deleterious effects after stroke. Nevertheless, the other two phenotypes contribute about $10 \%$ of monocytes, which play a beneficial role in patients with stroke [12]. Therefore, the comprehensive effects of peripheral blood monocytes reflect the roles of classical monocytes during the study of monocytes as a whole. Given the deleterious effects of classical monocytes and the possible neuroprotective effect of eosinophils [5, 10-12], our study found the lower EMR on admission was associated with higher risk of 3-month poor functional outcome in patients with AIS.

The main strength of our study is that the clinical information and blood samples of all patients were collected in a prospective fashion with a relatively large sample size.

In addition, we provided a comprehensive study for the eosinophils and monocytes by combining the two into a novel biomarker (EMR). Nonetheless, our current findings also have some limitations. First, the majority of acute critical patients were transferred to higher level hospitals due to the grass-roots hospitals nature of ours, which might result in the baseline NIHSS scores being relatively low with NIHSS median 3 (1-6) and existing deviations of the enrolled patients. Moreover, the baseline information of endovascular treatment which can impact stroke outcome was not been presented in the present study due to lack of this treatment in our hospital [22]. Thus, the findings might not generalize to other populations, particularly those with high NIHSS scores or experiencing endovascular treatment. Second, the predictive value of EMR for poor outcome is relatively low compared to other study (area under the curve: 0.6532 in stroke vs. 0.789 or 0.752 in patients with STelevation myocardial infarction) [9]. The reasons for the low predictive value may relate to the complicated roles of eosinophils and monocytes in ischemic insult, which have proinflammatory or anti-inflammatory properties [5, 10-12]. For example, we did not categorize the monocytes subpopulations which include $\mathrm{CD} 14^{\text {high }} \mathrm{CD} 16^{-}$, $\mathrm{CD} 14^{\mathrm{dim}} \mathrm{CD} 16^{+}$and $\mathrm{CD} 14^{\text {high }} \mathrm{CD} 16^{+}$phenotypes [12]. The other reason may relate to the timing chose for the EMR analysis, because it is unknown whether EMR is a dynamic variable as is the case with NLR [3]. Further studies are needed to investigate whether EMR is a dynamic variable and whether the subsequent EMR provide a greater predictive value for poor outcome than the baseline EMR. Third, we neither explored the mechanisms by which eosinophils and monocytes affected the neurovascular unit damage nor investigated what factors regulated the changes of eosinophils and monocytes after 
ischemic strokes in animal studies. These are going to be the focus of our next work, especially exploring the role of eosinophils in AIS and its mechanism.

\section{Conclusions}

In summary, we found that lower EMR on admission was associated with higher risk of 3-month poor functional outcome, indicating that EMR may be a potential prognostic biomarker for AIS. Further studies from other samples of patients with AIS are needed to validate our results.

\section{Supplementary Information}

The online version contains supplementary material available at https://doi. org/10.1186/s12868-021-00610-x.

Additional file 1: Table S1. Demographic and clinical characteristics of included and excluded patients. Table S2. The collinearity screening of baseline characteristics. Table S3. Associations of covariates with poor outcome $(\mathrm{N}=521)$. Table S4. The adjusting roles of potential confounders on the estimates of EMR on poor outcome. Table S5. The selected covariates. Figure S1. Flow chart of patient cohort. Figure S2. Prognostic value of eosinophil-to-monocyte ratio (EMR) in patients with ischemic stroke.

\section{Abbreviations}

AIS: Acute ischemic stroke; AUC: Area under the curve; Cl: Confidence interval; EMR: Eosinophil-to-monocyte ratio; GAM: Generalized additive models; IDI: Integrated discrimination improvement; IQR: Interquartile range; IV rtPA: Intravenous recombinant tissue plasminogen activator; mRS: Modified Rankin Scale; NIHSS: National Institutes of Health Stroke Scale; NRI: Net reclassification improvement; OR: Odds ratio; ROC: Receiver operating characteristic; TOAST: Trial of ORG 10172 in Acute Stroke Treatment.

\section{Acknowledgements}

Not applicable.

\section{Authors' contributions}

SHY, YL, CYZ and ZLG contributed to the concept and rationale for the study. SHY and $Y L$ were responsible for the first draft. CYZ and ZLG contributed statistical analyses. TZ, CRH, YF, QZ, FYZ and HH performed the data collection. CYZ and ZLG contributed to the first revision. All authors read and approved the final manuscript.

\section{Funding}

This study was supported in part by the National Natural Science Foundation of China (No. 81801154) and Suzhou City People's Livelihood Science and Technology Project (SYS201722, SYS2017051)

\section{Availability of data and materials}

The datasets used and/or analyzed during the current study are available from the corresponding author on reasonable request.

\section{Declarations}

\section{Ethics approval and consent to participate}

The study was carried out according to the Declaration of Helsinki and the Guideline for Good Clinical Practice. The study protocol was approved by the institutional Human Research Ethics Committees of Suzhou Integrated Traditional and Western Medicine Hospital, and written informed consent was obtained from all participants or their authorized relatives.

\section{Consent for publication}

Not applicable.

\section{Competing interests}

The authors declare that they have no competing interests.

\section{Author details}

${ }^{1}$ Department of Encephalopathy, Suzhou Hospital of Integrated Traditional Chinese and Western Medicine, Suzhou 215101, China. ${ }^{2}$ Department of Neurology and Suzhou Clinical Research Center of Neurological Disease, The Second Affiliated Hospital of Soochow University, No. 1055, Sanxiang Road, Suzhou 215004, Jiangsu, China.

Received: 4 July 2020 Accepted: 7 January 2021

Published online: 05 February 2021

\section{References}

1. Kollikowski AM, Schuhmann MK, Nieswandt B, Müllges W, Stoll G, Pham M. Local leukocyte invasion during hyperacute human ischemic stroke. Ann Neurol. 2020;87(3):466-79.

2. Semerano A, Laredo C, Zhao Y, Rudilosso S, Renú A, Llull L, et al. Leukocytes, collateral circulation, and reperfusion in ischemic stroke patients treated with mechanical thrombectomy. Stroke. 2019;50(12):3456-64.

3. Guo Z, Yu S, Xiao L, Chen X, Ye R, Zheng P, et al. Dynamic change of neutrophil to lymphocyte ratio and hemorrhagic transformation after thrombolysis in stroke. J Neuroinflamm. 2016;13(1):199.

4. Guo Z, Yu S, Chen X, Zheng P, Hu T, Duan Z, et al. Suppression of NLRP3 attenuates hemorrhagic transformation after delayed rtPA treatment in thromboembolic stroke rats: involvement of neutrophil recruitment. Brain Res Bull. 2018;137:229-40.

5. Jucevičiūtè N, Mikužis P, Balnytè R. Absolute blood eosinophil count could be a potential biomarker for predicting haemorrhagic transformation after intravenous thrombolysis for acute ischaemic stroke. BMC Neurol. 2019;19(1):127.

6. O'Connell GC, Chang JHC. Analysis of early stroke-induced changes in circulating leukocyte counts using transcriptomic deconvolution. Transl Neurosci. 2018;9:161-6.

7. Zhao HM, Qin WQ, Wang PJ, Wen ZM. Eosinopenia is a predictive factor for the severity of acute ischemic stroke. Neural Regen Res. 2019;14(10):1772-9.

8. Liberale L, Montecucco F, Bonaventura A, Casetta L, Seraceni S, Trentini $A$, et al. Monocyte count at onset predicts poststroke outcomes during a 90-day follow-up. Eur J Clin Invest. 2017:47(10):702-10.

9. Deng X, Wang X, Shen L, Yao K, Ge L, Ma J, et al. Association of eosinophilto-monocyte ratio with 1-month and long-term all-cause mortality in patients with ST-elevation myocardial infarction undergoing primary percutaneous coronary intervention. J Thorac Dis. 2018;10(9):5449-58.

10. Davoine F, Lacy P. Eosinophil cytokines, chemokines, and growth factors: emerging roles in immunity. Front Immunol. 2014;5:570.

11. Zierath D, Tanzi P, Shibata D, Becker KJ. Cortisol is more important than metanephrines in driving changes in leukocyte counts after stroke. J Stroke Cerebrovasc Dis. 2018;27(3):555-62.

12. Urra X, Villamor N, Amaro S, Gómez-Choco M, Obach V, Oleaga L, et al. Monocyte subtypes predict clinical course and prognosis in human stroke. J Cereb Blood Flow Metab. 2009;29(5):994-1002.

13. Sacco RL, Kasner SE, Broderick JP, Caplan LR, Connors JJB, Culebras A, et al. An updated definition of stroke for the 21st century: a statement for healthcare professionals from the American Heart Association/American Stroke Association. Stroke. 2013;44(7):2064-89.

14. Adams HP Jr, Bendixen BH, Kappelle LJ, Biller J, Love BB, Gordon DL, et al. Classification of subtype of acute ischemic stroke. Definitions for use in a multicenter clinical trial. TOAST. Trial of Org 10172 in acute stroke treatment. Stroke. 1993;24(1):35-41.

15. Liu Y, Zhao P, Cheng M, Yu L, Cheng Z, Fan L, et al. AST to ALT ratio and arterial stiffness in non-fatty liver Japanese population: a secondary analysis based on a cross-sectional study. Lipids Health Dis. 2018;17(1):275.

16. Zhu Z, Guo D, Zhong C, Wang A, Xie X, Xu T, et al. Serum Dkk-1 (Dickkopf-1) is a potential biomarker in the prediction of clinical outcomes among patients with acute ischemic stroke. Arterioscler Thromb Vasc Biol. 2019;39(2):285-93. 
17. DeLong ER, DeLong DM, Clarke-Pearson DL. Comparing the areas under two or more correlated receiver operating characteristic curves: a nonparametric approach. Biometrics. 1988;44(3):837-45.

18. Johnsen SH, Fosse E, Joakimsen O, Mathiesen EB, Stensland-Bugge E, Njølstad I, et al. Monocyte count is a predictor of novel plaque formation: a 7-year follow-up study of 2610 persons without carotid plaque at baseline the Tromsø study. Stroke. 2005;36(4):715-9.

19. Wang J, Ma L, Lin T, Li SJ, Chen LL, Wang DZ. The significance of eosinophils in predicting the severity of acute ischemic stroke. Oncotarget. 2017:8(61):104238-46.

20. Hori YS, Kodera S, Sato Y, Shiojiri T. Eosinopenia as a predictive factor of the short-term risk of mortality and infection after acute cerebral infarction. J Stroke Cerebrovasc Dis. 2016;25(6):1307-12.

21. Alba AC, Agoritsas T, Walsh M, Hanna S, lorio A, Devereaux PJ, et al. Discrimination and calibration of clinical prediction models: users' guides to the medical literature. JAMA. 2017;318(14):1377-84
22. Powers WJ, Rabinstein AA, Ackerson T, Adeoye OM, Bambakidis NC, Becker K, et al. Guidelines for the early management of patients with acute ischemic stroke: 2019 update to the 2018 guidelines for the early management of acute ischemic stroke: a guideline for healthcare professionals from the American Heart Association/American Stroke Association. Stroke. 2019;50(12):e344-418.

\section{Publisher's Note}

Springer Nature remains neutral with regard to jurisdictional claims in published maps and institutional affiliations.
Ready to submit your research? Choose BMC and benefit from:

- fast, convenient online submission

- thorough peer review by experienced researchers in your field

- rapid publication on acceptance

- support for research data, including large and complex data types

- gold Open Access which fosters wider collaboration and increased citations

- maximum visibility for your research: over $100 \mathrm{M}$ website views per year

At BMC, research is always in progress.

Learn more biomedcentral.com/submissions 